\title{
À propos d'une histoire des « Grammaires des Dames ». Réflexions théoriques et approches empiriques
}

Gabriele Beck-Busse

\section{(2) OpenEdition}

\section{Journals}

Édition électronique

URL : https://journals.openedition.org/dhfles/3121

DOI : $10.4000 /$ dhfles.3121

ISSN : 2221-4038

Éditeur

Société Internationale pour l'Histoire du Français Langue Étrangère ou Seconde

Édition imprimée

Date de publication : 1 juin 2012

ISSN : 0992-7654

\section{Référence électronique}

Gabriele Beck-Busse, «À propos d'une histoire des « Grammaires des Dames ». Réflexions théoriques

et approches empiriques », Documents pour l'histoire du français langue étrangère ou seconde [En ligne], 47-48 | 2012, mis en ligne le 01 janvier 2015, consulté le 25 mars 2023. URL : http://

journals.openedition.org/dhfles/3121; DOI : https://doi.org/10.4000/dhfles.3121

Ce document a été généré automatiquement le 25 mars 2023.

Tous droits réservés 


\title{
À propos d'une histoire des «Grammaires des Dames ». Réflexions théoriques et approches empiriques
}

\author{
Gabriele Beck-Busse
}

\section{Remarques préliminaires}

$1 \quad$ Il est évident que les conclusions qui suivent ne sont valables que pour les grammaires du corpus et pour les deux langues en question : le français et l'italien. Sera négligée la question de savoir si la situation se présente de la même façon pour, disons, les «Grammaires des Dames » de l'allemand, de l'anglais ou d'autres langues encore.

2 Conformément aux indications bibliographiques et à d'autres indices supplémentaires, le corpus est constitué d'une quarantaine de grammaires (37, pour être précise), dont à peu près deux tiers pour la langue française et un bon tiers pour l'italien (22 et 15). On pourrait parler d'un «corpus virtuel» vu qu'il n'existe pas, pour chaque indice, un exemplaire «palpable». Ceci dit, il faut préciser que le «corpus réel», le «corpus matériel » se réduit à 25 ouvrages, dont 15 pour le français et 10 pour l'italien.

3 S'y ajoute une bonne douzaine d'ouvrages (13) qui ne font pas partie du noyau du corpus mais qui, pour ainsi dire, "gravitent autour». Sans que ces ouvrages soient des «Grammaires des Dames» proprement dites, ils présentent, néanmoins, certains aspects qui renvoient au concept-clé, qui renvoient au concept de DAME ${ }^{1}$. Hors du noyau du corpus, il faut donc admettre une zone de transition dont il serait trop long de discuter les détails ici... 


\section{Questions théoriques}

\subsection{Choix du corpus - réflexions à propos des titres}

4 La détermination du corpus nous amène à la première des questions théoriques: comment délimiter, comment définir l'objet de l'étude ou, autrement dit: qu'est-ce qu'on entend, dans ce qui suit, par « Grammaire des Dames »?

5 La réponse paraît simple : fait partie du corpus tout ouvrage qui est une grammaire ou un manuel d'enseignement et qui porte dans son titre une formule telle que «des Dames », « pour les Dames » ou " à l'usage des Dames » (ou bien les équivalents italiens, anglais et allemands).

Quelles prémisses sont à l'origine d'un tel raisonnement? Quelles prémisses justifient une telle démarche? Je me limite, ici, à ne citer que Furetière; le fameux lexicographe témoigne de l'importance des titres en disant qu'un «beau titre est le vray proxenete d'un livre », proxénète, puisqu'il «fait faire le plus prompt débit » de l'objet qu'il affiche (Hoek (1981:3).

Mais le titre n'est pas seulement l'expression d'une stratégie mercantile, il est aussi l'expression de la conscience qu'il existe des types ou des genres d'ouvrage. Dans ce qui suit, je profite, entre autres, des travaux de Gérard Genette (1987: 82-85), qui explique comment un titre spécifique devient titre générique. Ce que Genette constate à l'égard des ouvrages littéraires se manifeste également en ce qui concerne les «Grammaires des Dames ». Jetons un coup d'œil sur les titres suivants :

The Ladies' $N$ e w Italian Grammar de Grimani (Londres 1788);

9 No u velle Grammaire italienne pour les dames d'Hennet (Paris 1790);

10 Ne w Italian grammar for ladies de Damiani (probablement Londres 1798);

11 Nouvelle Grammaire des Dames de Prevost-Desfourneaux (Nouv. éd., revue et corr. Paris 1805);

12 No u v ell e Grammaire des Dames d'Herrmann (Leipzig et Rostock 1809);

13 Cours analytique d'orthographe et de ponctuation, ou Nou velle Grammaire des Dames de Boinvilliers (Paris 1810).

14 De par la spécification "Nouvelle", les titres signalent qu'il faut considérer les ouvrages comme faisant partie d'une série ; qu'il existe un type, un genred'ouvrages qui partagent des traits en commun; qu'il existe des ouvrages qui, de certains points de vue, sont comparables.

15 La première tâche essentielle consistera à reconstruire les points de vue sous lesquels les ouvrages sont comparables (comparables par rapport à l'époque en question, c'està-dire par rapport aux XVII et XVIII ${ }^{e}$ siècles et à la première moitié du XIX ${ }^{\mathrm{e}}$ siècle).

16 Reprenant l'analyse des titres, il vaut la peine de regarder de plus près l'ouvrage de Prunay, publié à Paris en 1777. L'ouvrage est annoncé par un simple «Grammaire des Dames » - en lettres qui quêtent l'attention du lecteur et/ou de l'acheteur. Il ne suit ni titre secondaire ni sous-titre... Autrement dit : la page de titre présente «Grammaire des Dames » avec une certaine autonomie ; elle présente «Grammaire des Dames » en tant que formule auto-suffisante pour afficher l'ouvrage en question. Il est évidemment superflu d'expliciter à quoi « Grammaire des Dames » se réfère - tout le monde semble 
en être au courant - et l'arrangement (typo-)graphique illustre à quel degré, en 1777, l'épithète « des Dames » est, à Paris, chose naturelle².

Comme résumé de ces quelques réflexions titrologiques, il parait évident qu'au cours du XVIII ${ }^{e}$ siècle «Grammaire des Dames » devient générique. Le titre générique indique que l'ouvrage en question fait partie d'une série d'ouvrages commensurables condition indispensable pour une analyse comparée bien fondée.

Il faut aussi rappeler que le choix d'un titre n'est ni gratuit, ni arbitraire. En répondant aux besoins et aux espoirs de l'époque, le titre tient compte des exigences et des expectatives du côté de la production (auteur, éditeur) et du côté de la réception (public, audience, dédicataires etc.) ${ }^{3}$.

19 Les exigences et les expectatives sont, pour leur part, les reflets de la mentalité de l'époque ; elles aussi (comme DAME) sont des "constructa» ( $c$. Berger et Luckmann 1980). Ces «constructa» font partie des facteurs qui déterminent le choix du titre, comme l'explique Hoek. Tout comme Genette, Hoek se réfère aux Belles-Lettres mais, comme nous venons de le voir, les choses valent également pour les grammaires et les manuels d'enseignement. Hoek (1981: 3 ; c'est moi, qui souligne) dit :

[...] c'est au niveau du titre qu'on peut repérer le discours (ou la parole) social(e)

[...], où se croisent un langage romanesque (ou fictionnel) [ou bien grammatical], un

langage publicitaire et un langage idéologique, propres à une époque déterminée.

Il se pose, donc, la question suivante : quels sont les aspects pertinents, pertinents en ce qui concerne la commensurabilité des éléments de la série et pertinents en ce qui concerne « l'idéologie » de l'époque en question?

\section{2. À propos de 'Dame'}

Pour aborder ce point, il semble nécessaire d'étudier de plus près le concept de 'Dame'. C'est la seule voie pour avoir une idée plus nette de ce que, à l'époque, on qualifie de "Grammaires des Dames"; la seule voie pour mieux savoir ce que nous devons, nous aujourd'hui, entendre par ce titre, qui nous suggère, à nous, peut-être d'autres associations que celles qu'il suggérait autrefois. L'approche qui part de l'analyse du concept de 'Dame' semble être la seule possible pour avoir au moins la chance d'arriver à une analyse et une interprétation adéquates, appropriées et pertinentes sans courir le risque de succomber à l'anachronisme.

En 1774, le Journal des Dames, dans la recension d'une Physique des Dames, critique le fait que le titre ait été choisi uniquement pour des raisons publicitaires vu que l'ouvrage en question ne correspond pas aux expectatives que suggère la spécification «des Dames ». Ces expectatives, peut-on préciser, portent et sur la forme et sur le contenu de l'ouvrage. Le Journal des Dames (juillet 1774 : 76-77 ; c'est moi, qui souligne) dit :

On pourroit se demander pourquoi l'auteur de cet ouvrage l'a plutôt intitulé, la Physique des Dames, que la phisique des jeunes gens, des colleges, \&c. Il a cru sans doute le rendre plus intéressant par ce titre, \& se procurer les suffrages d'un sexe qui sait, quand il veut, donner tant de crédit \& de vogue à ce qu'il protege.

23 Le passage fait allusion à une certaine inflation, à un certain abus de titres tels que «Physique, Botanique, Logique, Rhétorique, Poétique, Grammaire... des Dames ». Cette inflation, cette mode, cet abus ne sont que le reflet du fait que l'on considère le marché comme susceptible d'une formule qui, de par l'épithète « des Dames », fait de l'ouvrage 
le «plus prompt débit » (pour reprendre les paroles de Furetière). Mais le passage nous apprend autre chose encore: le terme de 'Dame' évoque certaines qualités, qualités, que l'ouvrage en question, une certaine Physique des Dames, évidemment ne présente pas. C'est justement pour cela qu'on lui reproche de porter son titre à tort !

Il ne va pas de soi de trouver des indications qui témoignent explicitement que 'Dame' signifie plus que le rapport direct à un public féminin. Le passage suivant est d'autant plus précieux qu'il arrive assez rarement qu'on explicite ce qui est sous-entendu, ce qui fait partie du savoir partagé (cf. Schlieben-Lange, 1991: 309), ce qui appartient à la «mentalité ».

C'est une heureuse coïncidence que le libraire-éditeur d'un ouvrage, publié à la fin du $\mathrm{XVII}{ }^{\mathrm{e}}$ siècle, affirme explicitement que la référence à une 'Dame' est le résultat d'une intention bien déterminée. Autrement dit : on profite des associations que le concept de 'Dame' comporte pour signaler certaines particularités, certaines qualités, certaines caractéristiques de l'ouvrage. Par ailleurs, le libraire-éditeur de L'Éloquence du temps, enseignée à une Dame de qualité (1699 : [aVI ${ }^{\mathrm{r}-\mathrm{v}}$; c'est moi, qui souligne) adresse sa préface $\mathrm{Au}$ Lecteur (ce qui laisse supposer que le public qu'il envisage est des deux sexes). Voici ce qu'il dit :

[...] de quelque sexe que tu sois, \& quelque profession que tu ayes choisi[e], tu trouveras de l'utilité dans sa lecture [celle de l'Éloquence], l'Auteur l'ayant dediée à une Dame, af in de retrancher les termes ennuyeux de l'Ecole, \& accommoder son stile à la portée des gens, qui ont le moins de teinture de la Rhétorique.

Il est évident que, pour des raisons économiques et mercantiles, chaque éditeur tient à concevoir le public aussi large que possible. Ceci dit, j'aimerais focaliser l'attention sur la conjonction afin de. La finalité, liée à cette conjonction, laisse entendre que les idées autour du concept de 'Dame' entraînent, quasi automatiquement, certaines caractéristiques, certaines qualités. C'est cette implication automatique qui mérite, à mon avis, d'être explicitement mise en relief. De plus, les propos de l'éditeur sont encore plus détaillés. Sans scrupules, il expose ce à quoi on renvoie en «jouant la carte » de 'Dame': 'Dame' renvoie à un aspect du « style », c'est-à-dire au langage, et, en particulier, aux «termes ennuyeux », c'est-à-dire à la terminologie; et 'Dame' renvoie aux gens "qui ont le moins de teinture » de la matière en question, c'est-à-dire aux commençants.

27 Cet exemple suffira pour documenter que, aux XVII ${ }^{e}$ et XVIII ${ }^{e}$ siècles et au début du $\mathrm{XIX}^{\mathrm{e}}$ siècle, le terme de 'Dame' évoque beaucoup plus que le sexe et le rang social. En plus, l'exemple témoigne du fait que les titres du type « des Dames » ne poursuivent pas uniquement des buts commerciaux mais signalent au public des qualités spécifiques dont l'ouvrage en question dispose ou dont il tient à se parer - on se rappelle immédiatement la critique du Journal des Dames qui exige de la Physique des Dames certaines qualités pour qu'elle mérite, à juste titre, l'épithète « des Dames ».

Quelles sont les qualités que l'époque associe au concept de Dame? Il n'est pas étonnant que ce soit le premier des "Grammairiens des Dames " ${ }^{4}$, Annibale Antonini, qui éclaircit avec éloquence ce qu'il faut attendre d'une grammaire qui, dans son titre, fait référence (et révérence) "aux Dames». Dans ce cas aussi, nous assistons à une heureuse coïncidence : au moment de rédiger sa Grammaire italienne à l'usage des Dames, Antonini croit nécessaire d'expliquer au tout Paris en quels points sa deuxième Grammaire de 1728 se distingue de la première de 1726, intitulée Traité de la grammaire italienne, dédié à la Reine. Après neuf lignes introductives, le premier point de la préface 
à la Grammaire italienne à l'usage des Dames de 1728 traite de la question de savoir quel sera le public auquel l'ouvrage s'adresse. La place éminente et l'inclination de l'auteur à donner des explications détaillées sont le reflet de l'importance de la question au moment donné ${ }^{5}$. Voici les propos d'Antonini (1728: préface; sans pagination, sans signes de page) :

Je supposois dans mon premier Ouvrage, que tous mes Lecteurs, outre la connoissance du Latin, avoient du moins une legere teinture de la Langue Italienne ; un Livre executé sur un tel projet, ne devoit pas être du goût de tout le monde: Aussi la plûpart l'ont-ils negligé comme trop chargé de Regles \& de recherches. Je ne les en blâme pas ; à quoi bon faire une étude sérieuse, \& réguliere d'une langue que l'on n'apprend que pour s'amuser? C'est ainsi que voulant chercher un moyen pour instruire, j'en avois trouvé un pour déplaire.

J'ai composé cette nouvelle Grammaire pour ceux qui ne sçavent pas le Latin, \& qui n'ont pas la moindre connoissance de la Langue Italienne.

Dans cette vûe, j'ai retranché tout ce qui leur pourroit donner quelque embarras. On n'y trouvera que les Regles les plus nécessaires \& les plus simples, [é]noncées avec toute la clarté, la précision \& la brieveté, dont je suis capable. J'ai toûjours joint des exemples aux Regles, \& ces exemples ne sont que des phrases les plus aisées, \& les plus ordinaires dans la conversation.

Car j'ai travaillé pour ceux qui veulent apprendre sans peine, ou du moins avec une mediocre application. Une simplicité méthodique, \& dénuée de toute érudition, leur plaît, les amuse \& les instruit: Une Méthode trop sçavante \& trop etudiée les étonne, les rebute, \& ne les instruit point.

$\mathrm{Au}$ reste, cette Grammaire sera principalement à l'usage des Dames, quoique je sçache qu'il y en a parmi elles qui sont plus capables d'application que la plûpart des hommes, \& qu'en particulier elles ont beaucoup de pénétration pour apprendre les Langues.

Cependant je crus d'ailleurs qu'il falloit soulager leur peine, \& leur adoucir un travail, dont le succès peut m'être si glorieux. Rien ne seroit plus flatteur pour moi que de leur inspirer le goût d'une langue qui semble faite pour elles. [...]

Quoiqu'il en soit, si des hommes cherchoient aussi une Méthode aisée pour apprendre d'eux-mêmes l'Italien, \& sans beaucoup de fatigue, je crois qu'ils pourroient en toute assurance préferer la mienne à celle de Veneroni : car outre que j'ai sçû éviter quantité de fautes, où il est tombé, on trouvera dans ma Grammaire plusieurs Regles fort utiles, dont il ne fait aucune mention.

On ne se plaindra plus que je n'ai point donné de Dialogues; en voici plusieurs, \& puisqu'on les croit utiles aux commençans, je suivrai volontiers l'opinion du Public, même au préjudice de la mienne.

Ce sont les préfaces, les épîtres dédicatoires, les journaux littéraires, les réactions des concurrents et les critiques des contemporains, différentes remarques dispersées dans toute sorte de littérature, et, encore une fois, les titres qui nous fournissent des détails sur ce que nous devons associer à l'épithète «des Dames » : ils présentent le «mot de passe » DAMES dans des contextes révélateurs en explicitant des rapports et des relations significatifs et éloquents. Voici les résultats de la lecture : 1 . le terme de DAME évoque les représentants stéréotypiques, les représentants par excellence ou bien "prototypiques " d'un public qui peut être caractérisé comme suit et qui réunit en premier lieu «les Dames» et "les non-lettrés», mais qui lie «les Dames» aussi « aux Commençants » et à « la Jeunesse »; 2. l'auto-définition de ce public, dont «les Dames » ne sont que les représentants les plus voyants, recourt à l'idéal de la sociabilité, ce qui fait que l'érudit (pour ne pas dire le pédant) devient l'antagoniste par excellence ${ }^{6} ; 3$. le bon goût, qualité si naturellement assignée aux " Dames », est conféré au public entier et se transmet aux ouvrages dès qu'ils portent ce fameux « mot de passe » «Dames » : 
par ce geste, les ouvrages, c'est-à-dire contenu et présentation, réclament, pour eux, le bon goût que le concept de DAME comporte $; 4$. le public, représenté par «les Dames », cherche une méthode et une présentation qui se détachent nettement et indubitablement de l'érudition et qui, par cela, servent d'antidote à l'ennui ; 5. le public croit pouvoir attendre un style et un langage "anti-érudits", un langage et une présentation qui tiennent compte des caractéristiques sociables de la communication idéale, c'est-à-dire de la conversation soignée, présentation qui reconnaît dans les termes de l'art, dans le latin et le grec quelque chose qui mérite d'être mis à disposition - l'affinité aux idéaux de l'honnêteté est évidente ; 6 . en ce qui concerne la présentation, le public croit pouvoir attendre une élaboration, une composition qui soit agréable, divertissante, variée ; qui soit si claire et compréhensible que l'ouvrage puisse être étudié sans le secours d'un maître ; qui soit plutôt élémentaire, c'est-à-dire qui se limite à l'essentiel afin d'être brève, concise, pertinente; qui préfère le pratique au théorique, le concret à l'abstrait, en mettant l'accent sur l'application et l'applicabilité (exercices, dialogues ${ }^{8}$, vocabulaire, textes etc.) au lieu d'enseigner un système de règles stériles et arides et, par conséquent, peu utiles ; qui tienne compte de l'aspect « antiérudit ", aussi par rapport à la forme extérieure : que format et grosseur des volumes s'accordent aux idées qu'évoquent les concepts de 'Dame' et de 'Non-Lettré', c'est-àdire qu'exigent l'honnêteté, la sociabilité et le bon goût.

Une fois de plus, c'est un journal littéraire qui donne des indications précieuses : en 1728, lors de la parution de la grammaire d'Antonini, le Journal de Trévoux explique comment il faut lire l'épithète qui fait partie du titre. Tout en reprenant certaines tournures de la préface d'Antonini, le Journal de Trévoux (1728: 1565-1566) met en évidence, de son propre chef, le caractère synecdoquique ("par ce seul mot») de la formule « à l'usage des Dames " :

Elle [la Grammaire d'Antonini] est donc composée pour les personnes qui ne sçavent point le Latin, qui n'ont nulle connoissance de la Langue Italienne, qui veulent apprendre sans peine, \& qui n'ont à donner à ceci qu'une médiocre application ${ }^{10}$. L'Auteur exprime tout cela gracieusement \& heureusement par ce s e u $1 \mathrm{~m}$ ot, à l'usage des Dames.

Par ces quelques lignes, le Journal de Trévoux corrobore la lecture : aux XVII ${ }^{\mathrm{e}}$ et XVIII ${ }^{\mathrm{e}}$ siècles et au début du XIXe siècle, la formule "à l'usage des Dames " ne renvoie pas forcément au sexe mais plutôt aux non-lettrés, aux commençants, aux amateurs et dilettantes (c'est-à-dire aux dilettanti au sens vrai du mot) : à ceux qui « se délectent ", à ceux qui "se divertissent ", à ceux qui «s'amusent " (où "s'amuser » et "se divertir " signifient, selon le jargon de l'époque, que les personnes tiennent à ne pas faire profession de ce dont elles s'occupent). C'est aussi et surtout à cet aspect de nonprofessionnalité, d'anti-spécialisation qu'il faut penser en entendant parler de « divertissement » et de DAMES à l'époque qui nous intéresse.

\section{L'approche empirique}

\subsection{Réflexions préliminaires}

Après avoir dressé une liste des aspects que l'époque associe à l'épithète "des Dames ", le moment est venu d'établir les points de repère qui guideront l'analyse sérielle ${ }^{11}$. En disant sérielle, on souligne le caractère systématique de l'approche, systématique grâce à des paramètres constants et égaux pour l'ensemble ou un sous-groupe bien défini du 
corpus en question. Cette tâche semble, maintenant, moins difficile, car, ayant délimité les particularités que le concept de 'Dame' comporte (selon l'interprétation de l'époque), on a identifié le gros des paramètres qui détermineront l'analyse comparée.

L'analyse confrontera le concret (c'est-à-dire les ouvrages du corpus) à l'idéel (c'est-àdire aux idées à l'entour du concept de 'Dame') et elle le fera d'une façon systématique en soumettant la série aux mêmes paramètres. Pour des raisons d'homogénéité, il peut s'avérer raisonnable ou bien nécessaire de regrouper les éléments du corpus entier selon certains sous-critères dans le but de ne regrouper que ce qui est vraiment comparable (comparable dans un sens plus étroit que celui qui détermine l'établissement $d u$ corpus entier). Par la mise en question d'une correspondance automatique entre corpus en général d'une part et série d'ouvrages comparables d'autre part, l'analyse ne peut que gagner en rigueur. Autrement dit : en "structurant " la totalité du corpus, on reconnaît plus facilement les aspects systématiques.

Pour le corpus en question, cela signifie que les ouvrages se prêtent à être regroupés selon : 1. le lieu de publication / de réception (régions de langue française vs. régions de langue allemande) ; 2 . la langue enseignée (italien vs. français) ; 3 . le statut de la langue enseignée (langue maternelle vs. langue étrangère).

Il mérite d'être souligné qu'une démarche qui part des mots-clés de l'époque va du conceptuel à la réalisation et présente, à mon avis, plusieurs avantages : elle est fondée sur des paramètres qui ont été «re-construits " à partir du contexte de l'époque, paramètres qui courent beaucoup moins le risque d'être anachroniques; elle dispose d'un ensemble de paramètres qui guide l'analyse d'une manière systématique; elle offre la possibilité de reconnaître à quel degré la réalisation tient compte des caractéristiques idéelles (et idéales), d'évaluer les paramètres (en reconnaissant ceux parmi eux qui sont, dans ce contexte, les plus «importants " car les plus souvent " réalisés ", donc les plus fréquents) et de reconnaître plus facilement les particularités systématiques et les traditions spécifiques.

Tout en craignant d'être prolixe, on tient à répéter que le concept présente les caractéristiques d'une façon idéelle. Cela revient à dire qu'il faut distinguer entre conceptualisation et réalisation et qu'il sera utile de vérifier à quel degré l'un répond à l'autre, dans quelle mesure idée et réalisation cor-respondent. Voilà pourquoi, à mon avis, il s'impose de partir des idées pour interpréter les réalisations, c'est-à-dire les grammaires en question: en prenant la voie inverse, c'est-à-dire en partant des ouvrages sans avoir établi, d'emblée, un réseau de paramètres d'analyse, on est tenté de ne prendre en considération que ce qu'on retrouve car il n'est pas évident de pré-voir ce qui ne se présente pas (mais qui aurait pu ou dû y être) pour en faire l'analyse ; on est, à mon avis, plus facilement tenté d'interpréter les faits selon une perspective « moderne », c'est-à-dire anachronique. Partant de l'idéel, par contre, il est plus facile de reconnaître ce qui fait lacune ou ce qui est réalisé en "violant» les expectatives « communes »; il semble, donc, un peu plus facile d'adopter la perspective de l'époque en question. 


\subsection{L'analyse du corpus ${ }^{12}$}

\subsubsection{Chronologie}

La première trace d'une "Grammaire des Dames » remonte à 1688 et nous mène à Venise: dans sa Galerie française et italienne, dont il a été possible de consulter la cinquième «Chambre ", Louis de Pelenis $\left(1688:{ }^{*} 7^{\mathrm{r}}\right)$ annonce une Grammaire des Dames, qui constitue, selon l'auteur, l'introduction à la Galerie. Il n'a malheureusement pas été possible de localiser un exemplaire de ladite grammaire.

Le premier ouvrage accessible date de 1728: Annibale Antonini publie sa Grammaire italienne à l'usage des Dames à Paris.

C'est en 1744 seulement que commence la première phase d'" accumulation » ou de « condensation » : c'est en cette année que Steinbrecher publie sa Grammaire à Dresde ; suit Choffin avec la sienne à Berlin en 1747 ; Hase en publie une à Halle en 1750 ; Köster, ensuite, la sienne à Francfort en 1761; Thomas une autre à Ratisbonne en 1765; Giuliani, finalement, la sienne à Leipzig en 1768. On remarque que les lieux de publication sont exclusivement allemands. J'aimerais, en outre, ajouter que certaines des grammaires susnommées, surtout celles de Choffin et de Giuliani, sont rééditées (quelques-unes plusieurs fois) aux cours des années qui suivent.

En ce qui concerne la situation en France et à Genève, on constate que les « Grammaires des Dames » prennent leur essor justement dans les années où l'imprimerie et la librairie parisiennes se voient confrontées à de sérieuses difficultés ${ }^{13}$. Il ne paraît pas trop hasardeux de supposer un certain rapport entre le choix d'un titre captivant et séduisant et les tentatives de stimuler la vente.

41 Le premier ouvrage parisien, à part la grammaire d'Antonini de 1728, est celui de Bencirechi, en 1772, auquel suivent la grammaire de Prunay en 1777, celle de Nicolas Adam pour la langue française en 1779 et celle du même auteur pour la langue italienne en 1783. Le fil continue par Bencirechi avec ses Étrennes en 1783 également, par l'anonyme Grammaire de la Bibliothèque universelle des Dames et Barthélemy avec sa Grammaire en 1785, Secreti en 1787 et, de nouveau, Barthélemy avec sa Cantatrice en $1788^{14}$. Après la prise de la Bastille, en 1790, paraît, à Paris, la Nouvelle Grammaire italienne pour les Dames d'Albert-Joseph-Ulpien Hennet, personnage connu pour ses sympathies royalistes ${ }^{15}$. En 1792 - année du Manifeste de Brunswick (qui radicalisera la Révolution) - sera publiée encore, aux frais de l'auteur, la Méthode pour commencer l'étude de la langue italienne, à l'usage des Dames de Peppina Curioni ${ }^{16}$. Pour les années qui suivent, on ne trouve que deux indications, dont une pour l'étranger: en 1797, la Grammaire de Barthélemy est rééditée, non pas à Genève (lieu habituel des éditions), ni à Paris, mais en province, à Pont-de-Vaux, au nord de Mâcon ; en 1798, probablement, la New Italian grammar for ladies de Damiani voit le jour à Londres (cf. Alston (1987). Ces deux publications laissées de côté, les « Dames » reprennent le régime, tout doucement seulement, avec l'installation de Napoléon - ce qui ne paraît pas trop étonnant. Après la prise de la Bastille, pourtant, ce n'est évidemment pas un grammairien des dames qui est à l'ordre du jour, mais plutôt un grammairien patriote (c'est comment Boinvilliers apostrophe François-Urbain Domergue) ou un grammairien national (voilà Domergue à propos de Boinvilliers) ${ }^{17}$ !

Je me permets d'ajouter, entre parenthèses, quelques observations qui révèlent, encore une fois, comment les titres, même ceux des ouvrages "scientifiques", suivent les 
modes, voire les tendances politiques. À simple titre d'exemple, on peut renvoyer à $L a$ Science sans-culottisée de Decremps de 1794 (un traité d'astronomie) ${ }^{18}$, la Grammaire française républicaine de Bulard, publiée en $1795^{19}$, et la Grammaire des Sans-culottes d'Antoine Tournon, dont il n'existe que les traces du manuscrit et qui porte comme sous-titre Éléments de la langue nationale (cf. Guilhaumou, 1989: 129, 144). Un de nos "Grammairiens des Dames", à savoir Nicolas Adam (ou bien son éditeur), s'est vite aperçu que le vent a tourné : en 1790, la cinquième édition de la Grammaire française à l'usage des Dames de 1779 s'adapte au langage en vogue en ajoutant, sur la page de titre, la spécification "Grammaire Nationale ». À la page qui annonce la partie principale (Adam 1790 : 11), on combine, d'une manière assez habile, le vieux et le nouveau en disant : « GRAMMAIRE NATIONALE, FRANÇAISE UNIVERSELLE, A L'USAGE DES DAMES». Le contenu, par ailleurs, n'est pas soumis à un revirement significatif. Ces quelques remarques révèlent, une fois de plus, à quel degré les titres suivent la mode et sont l'expression de la mentalité - voilà un aspect de leur "proxénétisme » pour répéter l'heureuse formule de Furetière.

En résumant la chronologie des "Grammaires des Dames", on peut, pour les pays germanophones, parler d'une première phase d' "abondance " ou de "condensation " qui va de 1744 à 1768 (en tenant compte des rééditions, il faut l'élargir jusqu'en 1783) : dans cette période est publiée environ la moitié des ouvrages allemands. Une seconde phase de "condensation» comprend les années 1807 à $1811^{20}$. Quatre ouvrages et rééditions ne s'insèrent dans aucune des deux phases et paraissent plutôt tardivement : la réédition de la grammaire italienne de Giuliani, à Vienne en Autriche, en 1818; la grammaire française de Lange à Sulzbach, en 1824 ; la grammaire italienne de Schaub à Ulm, en 1824 ; la grammaire italienne de Kannegießer à Breslau, en 1836, et à Leipzig, en 1845 .

En ce qui concerne la France et Genève, on peut également distinguer deux phases importantes : entre 1772 et 1792 sont publiés douze ouvrages, c'est-à-dire à peu près deux tiers de l'ensemble des ouvrages publiés dans cette zone; entre 1803 et 1810 s'y ajoutent sept ouvrages et la Grammaire des Dames de Barthélemy est publiée pour la sixième fois ${ }^{21}$.

Les deux zones mises ensemble, on arrive à deux phases de condensation, qui vont de 1744 à 1792 et de 1803 à 1811.

Pays de langue allemande

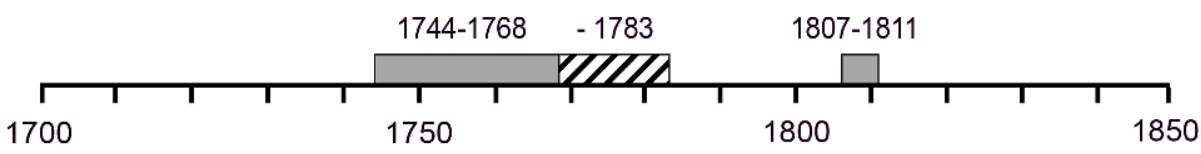

Paris et Genève 


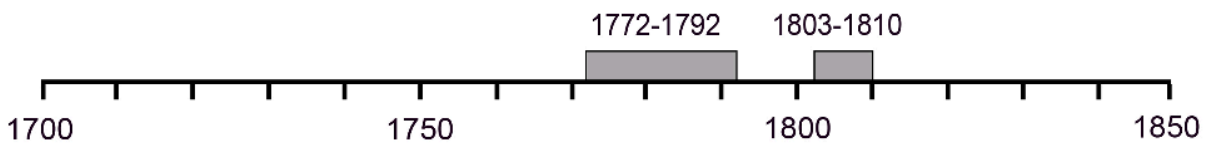

Paris, Genève et les pays de langue allemande ensemble

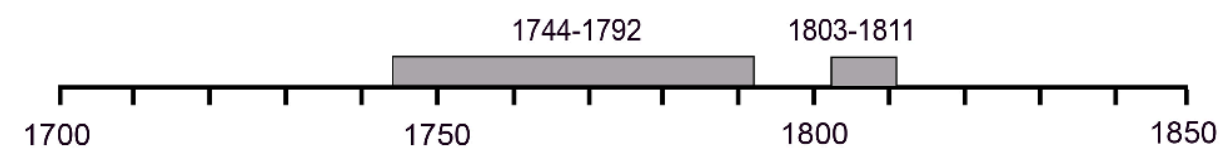

Phases de condensation

\subsubsection{Le côté de la matérialité : le format} ressortir le côté pratique) : presque deux tiers, à savoir 14 sur $23^{22}$, présentent, outre la description grammaticale au sens strict du mot (avec des préférences spécifiques selon la langue enseignée), 1. des dialogues, 2. un guide épistolaire, 3. une collection de proverbes, 4. des exemples de lecture (tels que: extraits de journaux, histoires et curiosités), 5 . des extraits ou des exemples d'auteurs modèles (comme, p. ex., un choix de lettres de personnages connus), 6. des extraits de poésie, 7. une explication du langage poétique ${ }^{23}, 8$. des chansons, 9 . des énigmes, 10 . des synonymes expliqués pour trouver le mot juste ${ }^{24}, 11$. une liste d'homophones, 12 . un recueil de prononciations vicieuses, 13. un traité d'orthographe, 14. un vocabulaire, 15. une introduction aux règles de la bienséance (cf. Choffin 1747, vol. 2).

\subsubsection{Le mode de présentation ${ }^{25}$}

Sous ce point s'entrecroisent le style (c'est-à-dire un langage anti-érudit, bienséant et sociable) et les genres littéraires à la mode. Pour témoigner de l'importance que l'époque attribue à la forme (et non seulement au contenu), j'aimerais répéter les 
propos du Journal de Trévoux (1720:118), qui, dans une de ses recensions, dit que «ce n'est qu'extraire à demi un ouvrage, si en rapportant ce qu'il contient, on ne conserve aussi quelque chose de sa maniére \& de son stile. » Le lemme qui résume, à mon avis à la perfection, l'analyse de "la manière » et "du style » peut être tiré de la Grammaire française de la Bibliothèque universelle des Dames (1785:3). Les auteurs expliquent : « Nous n'avons pas la prétention de mieux faire ; mais nous avons cru devoir faire autrement. »

En quoi consiste ce «faire autrement»? Il consiste à trouver une forme qui se détache de l'ordinaire du style grammatical pour enlever à la grammaire les épines et la sécheresse. En détail, cela revient à 1. donner un maximum d'explications (p. ex. une introduction aux termes grammaticaux, termes qui, en général, suivent la tradition latine) ${ }^{26} ; 2$. choisir une forme qui s'inspire des ouvrages périodiques (p. ex. celle des almanachs ${ }^{27}$; 3. présenter le contenu dans un langage qui tende vers l'oralité, qui s'inspire de l'entretien et qui coule le contenu dans le moule du style épistolaire ; 4. créer un cadre romanesque dans lequel s'insèrent les entretiens grammaticaux; cadre qui nous enlève souvent en Arcadie comme lieu idéal des entretiens sur la grammaire (on reconnaît l'importance de la littérature et surtout de celle pastorale) ${ }^{28}$; cadre qui permet à l'auteur d'expliquer pourquoi il se met au travail: non par un besoin pédantesque d'étaler son savoir, mais parce qu'on l'a instamment prié de communiquer ses précieuses connaissances dans la matière ; 5 . choisir une forme prosimétrique, c'està-dire insérer des parties rimées ou des chansons; les auteurs incitent leur public à apprendre la grammaire en chantant : par le frontispice à sa Grammaire en vaudevilles, Simonnin (1806) nous offre une belle image de son élève, ce qui, pour La Cantatrice grammairienne de Barthélemy (1788 : 224), signifie : «Les dames chanteront avec plaisir la chanson suivante qui vient à l'appui de la même regle ${ }^{29}$.

52 En ce qui concerne le souci de "faire autrement» il faut avouer que ce souci est beaucoup plus développé dans les régions de langue française qu'ailleurs. Dans les pays germanophones, c'est plutôt l'explication de la terminologie grammaticale latinisante, en combinaison avec un fort accent mis sur le côté pratique, qui s'inscrivent au premier plan des intentions des "Grammairiens des Dames ». En même temps, il vaut la peine de souligner que les traits susnommés ne sont pas limités aux "Grammaires des Dames »; on les retrouve aussi, soit dans la zone transitoire du corpus, soit dans des ouvrages qui ne font point allusion "aux Dames $»^{30}$. Néanmoins, ces traits sont inséparablement inhérents au concept de 'Dame' (dans la lecture de l'époque et dans notre contexte quasi-synonyme de 'Non-Lettré', ce qui porte à les combiner dans le couple synonymique 'Dame'-'Non-Lettré').

53 Ce qui justifie ce "faire autrement » n'est, à mon avis, que le vif souci de se détacher nettement du soi-disant " pédantisme ». Voilà la raison pour laquelle le commentaire de Brunot (vol. VII 1967 : 171) à propos de Barthélemy me paraît peu pertinent : à mon avis, les mots de Brunot ne tiennent pas suffisamment compte du fait que l'époque était contente, voire fière de son existence d'anti-érudit ou, mieux, d'anti-pédant. Ce n'est évidemment pas le pédantisme qui essaye de sourire et de plaisanter, mais, au contraire, c'est le Monde, qui tient à sourire à l'érudition et à plaisanter sur le pédantisme...

54 Le Monde a besoin de grammairiens qui rédigent dans un style et dans un langage «mondain " (où mondain doit être entendu dans le sens de "par rapport au Monde ", Monde avec majuscule) ${ }^{31}$. Le Monde a besoin de grammairiens qui maitrisent un langage qui respecte l'honnêteté et qui tienne compte de la sociabilité, dont la conversation 
soutenue est la forme d'échange par excellence. Le "lettré " aussi, surtout sous la forme du "philosophe $»^{32}$, est censé s'adapter à la conversation soutenue pour son échange culturel avec le Monde. Voilà comment s'explique l'affinité entre la littérature «des Dames» et l'oralité et il ne faut pas oublier que ce sont «les Dames» qui incarnent l'idéal de la sociabilité à la perfection.

\section{Conclusion}

Ce ne sont pas « les Dames » en tant que personnes qui sont en jeu ici. Ce qui intéresse, ce sont les idées que le concept de 'Dame' évoque (selon la lecture de l'époque en question). Ces idées expliquent les particularités d'une littérature qui se sert de l'épithète "à l'usage des Dames » pour signaler son opposition à l'érudition et au pédantisme. Il serait, par conséquent, erroné de penser à une littérature spécifiquement féminine, à une littérature pour les femmes ${ }^{33}$.

Dans notre contexte, il faut penser à une littérature qui fait appel aux idées autour du concept de 'Dame' dans le but (nous nous rappelons le fameux « afin de » de l'éditeur de l'Éloquence) d'afficher certaines qualités qui sont les bienvenues chez les "non-lettrés » en général, c'est-à-dire chez ceux qui ne savent pas le latin (ou n'en savent pas assez) et qui, vu l'affinité entre l'enseignement du latin et l'enseignement de la grammaire, peuvent être dénommés également "non-grammatisés "; certaines femmes en font partie - ainsi que les commençants, la jeunesse, et, évidemment, bien d'autres encore.

Vers la fin de l'époque en question, un auteur italien, Antonio Scoppa (1808: 8), caractérise sa "Grammaire des Dames » en disant: "Simplicité, clarté et précision, trois objets que je me propose dans ce petit ouvrage qui par ces raisons peut bien mériter le titre de Grammaire italienne pour les Dames ». Par simplicité, clarté et précision Scoppa fait référence au contenu de l'ouvrage ; il fait référence au cognitif, à la raison, à l'intellect et à l'entendement. Ceci est d'autant plus remarquable que, d'habitude, « les Dames" ne figurent pas du côté du cognitif, de la raison, de l'intellect et de l'entendement mais des sentiments, de la sensibilité, des émotions, de l'esthétique et des questions de goût. Simplicité, clarté, précision et 'Dame' ne s'accordent pas d'une manière im-médiate; les trois qualités s'accordent avec le concept de 'Dame' d'une manière in-directe et médiate : à travers l'affinité que l'époque conçoit entre 'Dame' et 'Non-Lettré' ; affinité qui se fait par rapport au contenu, au cognitif, à l'entendement ; affinité qui, enfin, suggère le jumelage 'Dame'-'Non-Lettré'.

L'aspect qui distingue 'Dame' de 'Non-Lettré' ne se trouve pas dans le champ intellectuel ( $\mathrm{du}$ côté du contenu) mais sur dans le champ social (du côté de la communication). C'est dans le champ de la pragmatique que 'Dame' l'emporte sur 'NonLettré': la sociabilité, qu'on accorde stéréotypiquement à 'Dame', fait que 'Dame' excelle à marquer l'antagonisme à 'Érudit' et à 'Pédant'. Le concept de 'Non-Lettré', par contre, ne renvoie pas à l'idéal de sociabilité ; 'Non-Lettré' ne peut pas faire ressortir l'aspect sociable du mode de communication mais se limite à mettre en relief le manque de connaissances de la langue latine (et de la grammaire). Autrement dit: tandis que 'Non-Lettré' marque le manque de grammatisation tout court (en laissant de côté l'aspect pragmatique, si important pour communiquer le contenu grammatical d'une manière adéquate), 'Dame' ne marque pas seulement le manque de grammatisation mais y ajoute l'aspect pragmatique: celui de communiquer la grammaire selon les 
règles du Monde. Et voilà la réconciliation avec ce qu'on attribue communément «au sexe » : la prédisposition à briller dans la communication, la faculté de "s'accommoder à la compagnie ", la fine sensibilité dans les relations sociales.

59 Bien que 'Dame' ne renvoie pas directement "au sexe ", cela n'implique pas que les études sur les «Grammaires des Dames " perdent leur intérêt pour les études sur l'identité de genre (gender identity). Bien au contraire : il est évident que le genre (gender) entre ici dans le vrai sens du mot puisqu'il n'est pas question du sexe tout court. Il est question de conceptualisations, il est question d'idées reçues autour d'un terme ('Dame') qui réunit aspects de sexe (important pour les recherches sur le genre), rôle dans la société... etc. etc. 

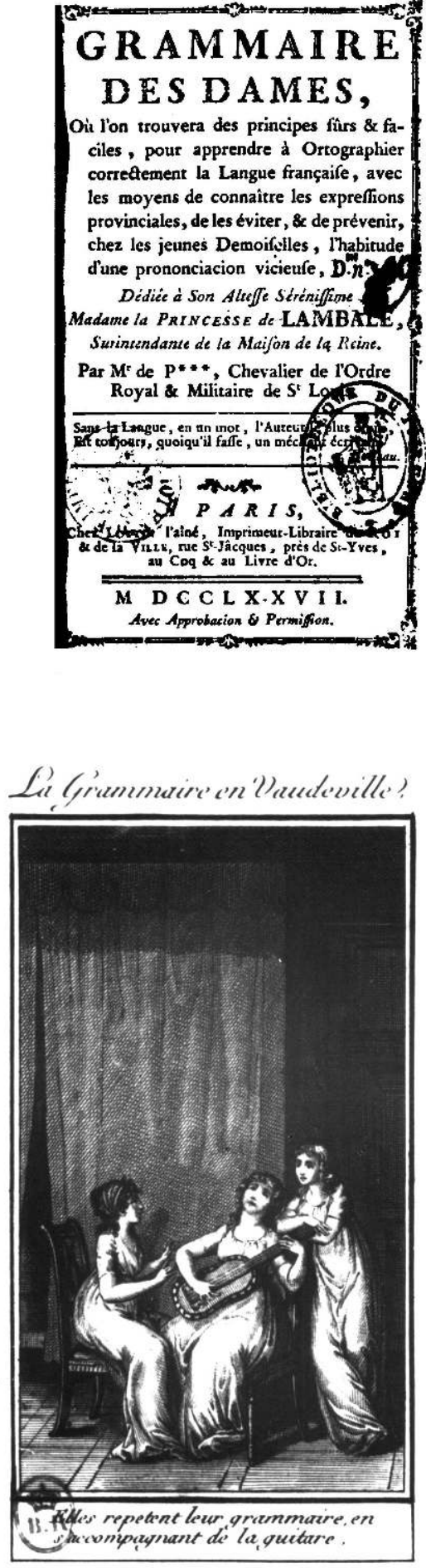

Simonnin. La Grammaire en vaudevilles. Paris, 1806.

60 «Les dames chanteront avec plaisir la chanson suivante qui vient à l'appui de la même regle » (Barthélemy, La Cantatrice grammairienne, $1788: 224$ ) 


\section{BIBLIOGRAPHIE}

ADAM, Nicolas (1779). La Vraie Maniere d'apprendre une langue quelconque, vivante ou morte, par le moyen de la langue françoise ; ouvrage divisé en plusieurs parties. $1^{\circ}$. Grammaire françoise à l'usage des Dames, servant de base à toutes les autres langues. $2^{\circ}$. Grammaire italienne.

$3^{\circ}$. Grammaire latine. $4^{\circ}$. Grammaire angloise. $5^{\circ}$. Grammaire allemande. $6^{\circ}$. Grammaire, \&c. \&c. \&c. $\mathrm{I}^{\mathrm{re}}$ partie. Paris : Morin.

ADAM, Nicolas (1783). Grammaire italienne, à l'usage des Dames. Paris : Morin.

ADAM, Nicolas ( ${ }^{5} 1790$ [1779]). La Vraie Manière d'apprendre une langue quelconque. Première partie. Grammaire nationale. Paris : Morin.

ALGAROTTI, Francesco (1737). Il Newtonianismo per le Dame ovvero dialoghi sopra la luce e i colori. Naples : s. éd.

ALSTON, R. C. (1985, 1987). A bibliography of the English language from the invention of printing to the year 1800. Vol. 12. Part 1: The French Language. Part 2: The Italian, Spanish, Portuguese and Romansh Languages. Otley: Smith Settle.

ANTONINI, Annibale (1726). Traité de la grammaire italienne, dedié à la Reine. Paris : Lottin. ANTONINI, Annibale (1728). Grammaire italienne à l'usage des Dames. Paris : Rollin/Bordelet. AYRES-BENNETT, Wendy (1990). « Women and Grammar in seventeenth-century France », Seventeenth Century French Studies, 12, 5-25.

BAADER, Renate (1986). Dames de Lettres. Autorinnen des preziösen, hocharistokratischen und 'modernen' Salons (1649-1698): Mlle de Scudéry - Mlle de Montpensier - Mme d'Aulnoy. Stuttgart : Metzlersche Verlagsbuchhandlung.

BARBIER, Antoine-Alexandre $\left({ }^{3} 1872-1879 ; 1889\right)$. Dictionnaire des ouvrages anonymes. Paris : Paul Daffis. Supplément par Gustave Brunet. Paris : Féchoz.

BARTHÉLEMY, Louis (1785). Grammaire des Dames, ou Nouveau Traité d'orthographe françoise. Genève : Barde.

BARTHÉLEMY, Louis (1788). La Cantatrice Grammairienne, ou l'art d'apprendre l'orthographe française seul, sans le secours d'un maître, par le moyen des chansons érotiques, pastorales, villageoises, anacréontiques, \&c. Ouvrage destiné aux Dames. Genève : s. éd.

BECK-BUSSE, Gabriele (1994). « Les 'femmes' et les 'illitterati'; ou : la question du latin et de la langue vulgaire », Histoire Epistémologie Langage, 16/II, 77-94.

BECK-BUSSE, Gabriele (1999). « La grammatica in Arcadia: “The French Garden for English Ladyes and Gentlewomen to walke in" di Peter Erondell (Londra 1605) », in Silvana Seidel Menchi, Anne Jacobson Schutte \& Thomas Kuehn éds, Tempi e spazi di vita femminile tra medioevo ed età moderna. Bologne : Il Mulino, 175-191.

BECK-BUSSE, Gabriele (2001). « Grammar in Arcadia », in Anne Jacobson Schutte, Thomas Kuehn \& Silvana Seidel Menchi eds, Time, Space and Women's Lives in Early Modern Europe. Kirksville : Truman State University Press, 29-40.

BELLEGARDE, l'Abbé (1727). The Praise of Women. In Answer to this Question; Whether the Company of Women is useful, or hurtful, to young Gentlemen at their first Setting out in the World? Londres : s. éd. 
BENCIRECHI, L'Abbé (1772). Leçons hebdomadaires de la langue italienne à l'usage des Dames. Paris : Veuve Ravenel/Fetil.

BENCIRECHI, L’Abbé (1783). Étrennes italiennes, présentées aux Dames. Paris : Dénos/Molini. BERGER, Peter L. \& LUCKMANN, Thomas (1980 [1966]). Die gesellschaftliche Konstruktion der Wirklichkeit. Eine Theorie der Wissenssoziologie. Francfort-sur-le-Main : Fischer Taschenbuch Verlag.

Bibliothéque universelle des Dames. Troisième classe. Mélanges. Paris : s. éd., 1785-1789. Tome 1 : Grammaire françoise (1785), tome 2 : Traité de l'ortographe (1785).

BOINVILLIERS, Jean-Étienne-Judith Forestier, dit (1810). Cours analytique d'orthographe et de ponctuation, ou nouvelle grammaire des Dames. Paris : Delalain.

BRUNOT, Ferdinand (1905-). Histoire de la langue française des origines à 1900. Paris : Colin. BUSSE, Winfried (1981). « Domergue, grammairien patriote », in Jürgen Trabant, Logos semantikos. Studia linguistica in honorem Eugenio Coseriu 1921-1981. Vol. 1: Geschichte der Sprachphilosophie und der Sprachwissenschaft. Berlin etc. : de Gruyter/ Gredos, 371-384. BUSSE, Winfried \& DOUGNAC, Françoise (1992). François-Urbain Domergue. Le grammairien patriote (1745-1810). Tübingen : Narr.

CHERVEL, André (1977). ... et il fallut apprendre à écrire à tous les petits Français. Histoire de la grammaire scolaire. Paris : Payot.

CHOFFIN, David Étienne (1747). Nouvelle Grammaire à l'usage des Dames, et des autres personnes qui ne savent pas de latin. Tome II. Berlin : Haude et Spener.

CONDILlaC, l'Abbé de (1986 [1775]). Cours d'étude pour l'instruction du Prince de Parme. Tome premier. Grammaire. Stuttgart-Bad Cannstatt : Frommann-Holzboog.

CURIONI, Peppina (1792). Méthode pour commencer l'étude de la langue italienne, à l'usage des Dames. Paris : L'Auteur.

DAMIANI, F. (1798?). A new Italian grammar for ladies. [Londres]. [Selon Alston 1987: \# 109]

DAVIS, Natalie Zemon (1979). «L'Imprimé et le peuple », in Natalie Zemon Davis, Les cultures du peuple. Rituels, savoirs et résistances au $16^{\mathrm{e}}$ siècle. Paris : Aubier-Montaigne, 308-365.

Dictionnaire de biographie française (1933-). J. Balteau, M. Barroux, M. Prevost etc. éds. Paris : Letouzey et Ané.

L'Éloquence du temps, enseignée à une Dame de qualité selon les regles d'une rhetorique aisée \& galante (1699). s. l. [Souvent attribué à Leven de Templeri ; probablement à Paris chez J. Leonard] ENGELMANN, Wilhelm (1842). Bibliothek der neueren Sprachen oder Verzeichniß der in Deutschland besonders vom Jahre 1800 an erschienenen Grammatiken, Wörterbücher, Chrestomathien, Lesebücher und anderer Werke. Leipzig : Engelmann.

ERSCH, Johann Samuel (1969-1970 [1793, 1799-1800, 1807]). Allgemeines Repertorium der Literatur. Berne : Herbert Lang.

FONTENELLE, Bernard Le Bovier de (1966 [1686]). Entretiens sur la pluralité des mondes. Paris : Didier.

GENETTE, Gérard (1987). Seuils. Paris : Éditions du Seuil. GIULIANI, Francesco Savero (1768). Gramatica toscana italiana per le Dame. Leipzig : Sommer. 
GOODMAN, Dena (2002). «L'ortografe des dames : Gender and Language in the Old Regime ", French Historical Studies, 25/2, 191-223.

GRIMANI, Gasparo (1788). The Ladies['] New Italian Grammar. Londres : Printed by W. Smith/The Author.

GUILHAUMOU, Jacques (1989). La langue politique et la Révolution française. De l'événement à la raison linguistique. Paris : Méridiens Klincksieck.

HASE, Christian Gottfried (1750). Philosophische Anweisung zur französischen, italiänischen und englischen Sprache. Halle : Gebauer.

HENNET, Albert-Joseph-Ulpien (1790). Nouvelle Grammaire italienne pour les dames. Paris : s. éd. [selon Barbier: s. v. nouvelle et Mormile : 269]

HERRMANN, G. F. (1809). Neue erleichterte und faßliche Französische Sprachlehre zum practischen Unterricht/Nouvelle Grammaire des Dames. Rostock/Leipzig : Stillersche Buchhandlung.

HOEK, Leo H. (1981). La marque du titre. Dispositifs sémiotiques d'une pratique textuelle. La Haye/ Paris/New York : Mouton.

Journal de la langue françoise, soit exacte, soit ornée. Genève : Slatkine, 1978 [1784-1788, 1791-1792].

Journal des Dames, dedié a Madame La Dauphine. Paris : Lacombe.

Journal de Trévoux. Genève : Slatkine, 1968-1969 [1701-1767].

KANNEGIESSER, Karl Ludwig ( $\left.{ }^{2} 1845[1836]\right)$. Italienische Grammatik nebst Lesebuch und Wörterverzeichniß für Anfänger und Geübtere und vorzüglich auch für Damen; mit einer Geschichte der italienischen Literatur. Leipzig : Fr. Hentze.

KÖSTER, Henrich Martin Gottfried (1761). Anleitung zur Französischen Sprache zum Gebrauch des Frauenzimmers, und anderer welche kein Latein verstehen. Frankfurt/Leipzig : Raspe.

KOTZEBUE, August von ( ${ }^{3} 1843$ [1804]). Erinnerungen aus Paris im Jahre 1804. Wien : Klang.

LALANDE, Joseph-Jérôme Le François de (1970 [1803]). Bibliographie astronomique ; avec l'histoire de l'astronomie depuis 1781 jusqu'à 1802. Amsterdam : Gieben.

LANGE, Moritz (1824). Vollständige französische Sprachlehre für Damen. Sulzbach : Seidel. MARTIN, Henri-Jean (21990 [1982-1986]). « Une croissance séculaire », in Roger Chartier \& HenriJean Martin, Histoire de l'édition française. Vol. II : Le livre triomphant. 1660-1830. Paris : Fayard/Cercle de la Librairie, 113-125 ; annexe : 125-127.

MINARD, Philippe (1989). Typographes des Lumières. Suivi des « Anecdotes typographiques » de Nicolas Contat (1762). Seyssel : Champ Vallon.

MORMILE, Mario (1989). L'italiano in Francia. Il francese in Italia. Storia critica delle opere grammaticali francesi in Italia ed italiane in Francia dal Rinascimento al Primo Ottocento. Turin : Meynier.

MORTUREUX, Marie-Françoise (1989). « “L'Astronomie Fontenellisée” ou : Science, Langage et Société dans l'episteme classique », in Brigitte Schlieben-Lange éd, Fachgespräche in Aufklärung und Revolution. Tübingen : Niemeyer, 81-114.

PELENIS, Louis de (1688). La Galerie françoise et italienne. Cinquieme chambre. Les complimens à la mode, divisez en trois leçons. Venise : Poletti. 
PHILIPON-DE-LA-MADELEINE, L. (1807). Grammaire des gens du Monde, ou la langue française enseignée par l'usage. Paris : Imprimerie de Richomme/Capelle et Renand.

PREVOST-DESFOURNEAUX, ? (1805). Nouvelle Grammaire des Dames, ou l'art d'apprendre le français sans maître. Paris : Châtel. [Selon Quérard]

PRUNAY, Mr. de (1777). Grammaire des Dames, où l'on trouvera des principes sûrs \& faciles, pour apprendre à ortographier correctement la langue française. Paris : Lottin l'aîné.

QUÉRARD, J.-M. (1964). La France littéraire, ou dictionnaire bibliographique des savants, historiens et gens de lettres de la France. Paris : G.-P. Maisonneuve \& Larose.

REINACH, Salomon (1913). Sidonie ou le français sans peine. Paris : Hachette.

SANSON, Helena (2011). Women, Language and Grammar in Italy, 1500-1900. Oxford : British Academy/Oxford University Press.

SCHAUB, J. Bt. (1824). Italienische Grammatik für Frauenzimmer. Ulm : Stettin. [Selon Ersch: \# 7063 et Engelmann: 221]

SCHLIEBEN-LANGE, Brigitte (1984). « Vom Vergessen in der Sprachwissenschaftsgeschichte. Zu den 'Ideologen' und ihrer Rezeption im 19. Jahrhundert », Zeitschrift für Literaturwissenschaft und Linguistik, 53/54, 18-36.

SCHLIEBEN-LANGE, Brigitte (1988). «Le ‘style féroce’ de Louis-Sébastien Mercier : l'écriture de l'inouï », in Dictionnaire des usages socio-politiques (1770-1815), 3, 133-150.

SCHLIEBEN-LANGE, Brigitte (1991). « Hermeneutik und Serie », in Brigitte Schlieben-Lange et al. éds, Europäische Sprachwissenschaft um 1800. Methodologische und historiographische Beiträge zum Umkreis der « idéologie ». Band 2. Eine Vortragsreihe im Rahmen des DFG-Projekts

« Ideologenrezeption ». Münster : Nodus, 307-318.

scopPA, Antoine/Antonio (1808). Grammaire italienne pour les Dames. Paris : Gérard.

SECRETI, Louis (1787). Grammaire italienne pour les Dames, et pour ceux qui n'ont pas appris le latin. Genève : s. éd. [« Et se vend chez le sieur Muzet »]

SEGLER-MESSNER, Silke (1998). Zwischen Empfindsamkeit und Rationalität. Der Dialog der Geschlechter in der italienischen Aufklärung. Berlin : Schmidt.

SIMONNIN, Antoine-Jean-Baptiste (An XIV/1806). La Grammaire en vaudevilles, ou lettres à Caroline sur la grammaire française. Paris : Barba.

STEINBRECHER, M. Gottfried (1744). M. Gottfried Steinbrechers leichte frantzösische Grammaire vor das Frauenzimmer. Dresde : Gerlachen.

THOMAS, Johannes/Jean (1765). L'Art d'aprendre la langue françoise en Allemagne. Die Kunst, die französische Sprache in Deutschland zu lernen; für das Frauenzimmer und auch für die der lateinischen Sprache wenig erfahrnen Mannspersonen. Ratisbonne : Englerth.

TIMMERMANS, Linda (1993). L'Accès des femmes à la culture (1598-1715). Un débat d'idées de Saint François de Sales à la Marquise de Lambert. Paris/Genève : Honoré Champion/Slatkine.

WAILLY, Noël-François de (1782). L'Orthographe des Dames, ou l'orthographe fondée sur la bonne prononciation, démontrée la seule raisonnable. Paris : Mérigot le jeune. 
ZANCAN, Marina (1983). « La donna e il cerchio nel Cortegiano di B. Castiglione. Le funzioni del femminile nell'immagine di corte », in Marina Zancan, Nel cerchio della luna. Figure di donna in alcuni testi del XVI secolo. Venise : Marsilio Editori, 13-56.

Prunay. Grammaire des Dames. Paris, 1777.

\section{NOTES}

1. Les petites capitales servent à mettre en relief le fait que l'on parle du concept (et non pas de la dénotation).

2. Ce qui suit le titre «Grammaire des Dames » est une espèce de compte rendu du contenu et aurait pu être introduit aussi par l'habituel contenant ... ou renfermant ...

L'exemple du manuel de Boinvilliers (Cours analytique d'orthographe et de ponctuation, ou Nouvelle Grammaire des Dames) est d'autant plus révélateur qu'il témoigne que, à l'époque de sa publication, c'est-à-dire en 1810, «Grammaires des Dames » sert de titre secondaire. Il ne semble pas exagéré d'interpréter ce titre secondaire non seulement comme titre alternatif, mais aussi comme indication de genre, comme indication générique ( $c f$. l'épithète nouvelle). Dans le cas de Boinvilliers, le titre secondaire « Nouvelle Grammaire des Dames » assume donc deux fonctions parallèles: celle de titre secondaire et celle d'indication générique. En ce qui concerne la titrologie, je renvoie sommairement à Genette (1987) et Hoek (1981).

3. En ce qui concerne la distinction entre public et audience, je renvoie à Davis (1979).

4. En parlant de «Grammairiens des Dames », je reprends une expression de Simonnin (1806: 49).

5. Malgré le succès des Entretiens sur la pluralité des mondes de Fontenelle, publiés, pour la première fois, en 1686, quarante ans plus tard, en 1728, les titres du type « (à l'usage) des Dames " ne sont apparemment pas encore aussi naturels qu'en 1777, quand la grammaire de Prunay sort des presses. Il vaut, en outre, la peine de rappeler que le Newtonianismo per le Dame d'Algarotti, ouvrage qui faisait éclat à l'époque, sera imprimé en 1737 seulement - ce qui n'empêche pourtant pas que l'ouvrage (ou des extraits) ne soient pas connus sous d'autres formes que celle d'un livre imprimé...

6. Cf. p. ex. Baader (1986: 215-217), Bellegarde (1727: 29-30) et Kotzebue (1843: 420-421).

7. Cf. Zancan (1983: 30-32), Ayres-Bennett (1990: 8-9 ; 1994b), Timmermans (1993: 152-168) et Segler-Meßner (1998 : 87-89) ; voir aussi Prunay (1777 : XXXI-XXXII).

8. On se souvient des propos d'Antonini dans la préface à sa Grammaire de 1728.

9. Les caractères italiques se trouvent dans l'original ; les caractères espacés sont les miens.

10. Aux XVII ${ }^{\mathrm{e}}$ et XVIII ${ }^{\mathrm{e}}$ siècles, médiocre recourt au juste milieu entre les extrêmes.

11. En ce qui concerne l'approche sérielle, l'approche biographique et l'étude approfondie, $c f$. Schlieben-Lange (1984, 1988, 1991).

12. Malgré, peut-être, quelque tendance à généraliser, il paraît opportun de rappeler que les conclusions qui suivront ne seront formulées que par rapport aux grammaires pour les langues française et italienne.

13. En ce qui concerne l'édition parisienne des années 70 et 80 du XVIII ${ }^{\mathrm{e}}$ siècle $c f$. Minard (1989 : 92) et Martin (1990).

14. Deux ouvrages d'Antonio Curioni ne peuvent être datés que selon les indications bibliographiques qui permettent de les localiser avant 1781 et 1788 respectivement.

15. À propos d'Hennet le Dictionnaire de biographie française (1933 sqq.: s.v. Hennet) précise : «Après la Révolution [la prise de la Bastille], il resta fidèle au roi et ne craignit pas de se faire remarquer en envoyant une Pétition à l'Assemblée nationale par Montaigne, Charron, Montesquieu et Voltaire, 1791, puis, surtout, par une Complainte de Louis XVI aux Français, chantée dans la première quinzaine de janv. 1793, mais interdite rapidement par la police. » 
16. Il n'a malheureusement pas été possible de révéler d'éventuels liens de parenté entre Antonio et Peppina Curioni.

17. Cf. le Journal de la langue française de Domergue (vol. 2, $1978: 125,154$ [2 avril 1791: 1 ; 23 avril 1791 : 117]) et, à propos de Domergue, Busse (1981) et, ensuite, Busse et Dougnac (1992).

18. Cf. Lalande (1970 [1803] : 631).

19. Cf. Guilhaumou (1989 : 139) ; la Bibliothèque nationale à Paris en possède un exemplaire de la deuxième édition (sans année).

20. $\mathrm{Vu}$ les tendances nationalistes qu'éveillent l'établissement de la Confédération du Rhin en 1806 et les guerres napoléoniennes, il paraît, sans doute, un tantinet étonnant que, justement en ces années-là, on réanime, à Leipzig, Rostock, Rudolstadt et Weimar (lieux de publication desdites grammaires), la mode des « Grammaires des Dames » pour enseigner la langue « du vainqueur» : le français.

21. "Après la lettre ", en 1913 (!), paraît encore un ouvrage qui reprend certaines des idées des Grammaires à l'usage des Dames (et du Grand Siècle) sans, pour autant, adopter un titre tel que "Grammaire des Dames ». Dans la préface à Sidonie ou le français sans peine, Salomon Reinach (1913 : avant-propos; sans pagination) explique : "Aucune langue ne doit plus que la nôtre à cette école de raffinement mutuel qu'est la conversation. Non pas la conversation des rues, des camps ou des collèges [...] mais celle des salons que fréquentent les deux sexes, berceaux de l'affabilité, de la gaîté contenue, des mœurs douces, où Mlle de Scudéri voulait [...] qu'un certain 'esprit de joie' s'alliât à 'l'esprit de politesse.' Il m'a semblé que je rendais encore hommage à l'aimable origine de la plus sociable des langues en l'enseignant, du mieux que j'ai pu, sur le ton des entretiens qui l'ont formée. »

22. Le nombre de 23 (réduction significative par rapport au nombre total des ouvrages, mentionnés dans la partie chronologique) s'explique par deux faits : 1 . l'analyse du contenu, du format etc. ne peut porter que sur les ouvrages accessibles (cf. la distinction entre " corpus réel » et «corpus virtuel», distinction de très peu d'importance, pourtant, pour établir une chronologie) ; 2. on est forcé de négliger les deux ouvrages de Bencirechi car l'auteur choisit, pour ses ouvrages, la structure leçon par leçon ce qui rend une répartition du contenu assez difficile. Secreti (1787), qui consacre $99,35 \%$ de sa grammaire italienne à la description grammaticale (153 sur 154 pages), figure (par généralisation) parmi les neuf ouvrages pour lesquels le côté de l'applicabilité ne porte, selon les apparences, aucun intérêt.

23. Voilà un aspect important dans l'enseignement de la langue italienne.

24. Les points 10 à 13 sont d'une extrême importance dans l'enseignement de la langue française aux Français(es) mêmes. En ce qui concerne le problème des homophones et de l'orthographe $c f$. aussi Chervel (1977: 46) : "Pour écrire correctement le français, il va donc falloir devenir grammairien. » En ce qui concerne le rôle (et l'importance) « des Dames » dans une réforme de l'orthographe $c f$. de Wailly (1782) et, plus récemment et pour une vue d'ensemble, Goodman (2002).

25. Voilà, à mon avis, l'aspect le plus intéressant, le plus complexe et le plus important pour expliquer le phénomène des «Grammaires des Dames » par rapport au concept central, celui de 'Dame'-'Non-Lettré'.

26. En ce qui concerne la soi-disant incompatibilité de 'Dames'-'Non-Lettrés' avec le latin et quant à leur présupposée affinité à la langue «vulgaire » (= volgare) $c f$. p. ex. Beck-Busse (1994).

27. Dans ses Étrennes italiennes, qui paraissent, d'ailleurs, chez un éditeur spécialisé dans l'édition des almanachs, Bencirechi $(1783, c f$. aussi les Leçons de 1772) se sert de cette forme pour joindre l'utile à l'agréable: l'ensemble est réparti en entités plus maniables et l'abonnement hebdomadaire présente l'aspect de nouveauté.

28. En ce qui concerne le rapport entre le discours grammatical et l'Arcadie $c f$. Beck-Busse (1999, 2001). 
29. Dans son Histoire de la langue française, Brunot (vol. VII 1967 : 171) dit à propos de la Grammaire des Dames de Barthélemy que c'est un ouvrage « où le pédantisme essaie de sourire, de plaisanter, de madrigaliser »; on y reviendra tout de suite.

30. On ne mentionnera que le Cours d'étude pour l'instruction du prince de Parme de Condillac (1986 [1775]), qui présente certains aspects d'oralité.

31. On pense tout de suite à la Grammaire des gens du monde, ou la langue française enseignée par l'usage de Philipon-de-la-Madeleine (1807).

32. Quant au " philosophe », cf. Mortureux (1989).

33. Pour une approche beaucoup plus élargie, à savoir la relation entre femmes (en tant que personnes) et grammaire/langue, $c f$. récemment Sanson (2011).

\section{RÉSUMÉS}

Les XVIIIe et XIXe siècles connaissent des ouvrages portant des titres tels que « Grammaire des Dames ", "Grammaire pour les Dames » ou bien "Grammaire à l'usage des Dames ». Une telle spécification implique un concept (DAME) qui renvoie à une entité sociale qui, elle, fait partie de l'idéologie à un moment donné. Dresser l'histoire des " Grammaires des Dames » signifie donc aborder la grammaire sous un angle plutôt socio-idéologique que grammatico-théorique. Les questions qui se posent seront les suivantes : Quels sont les aspects pertinents de l'angle socioidéologique ? Comment les repérer et les identifier ? Comment les interpréter et les intégrer dans une vue d'ensemble ? La partie empirique analysera 25 «Grammaires des Dames », dont 15 pour la langue française et 10 pour l'italien. Les ouvrages du corpus ont été publiés entre 1728 et 1845, en France et à Genève, dans les pays germanophones et à Londres. En se référant à quelques-uns des paramètres établis au cours des réflexions théoriques, il sera possible d'étudier : à quel degré les manifestations concrètes corroborent les résultats théoriques, à quel point on peut constater des traditions locales (la France et Genève d'une part, les pays germanophones d'autre part), à quel point le statut de la langue (maternelle ou étrangère) explique certaines caractéristiques, en quel sens l'enseignement de l'italien suggère d'autres contenus et suit différents chemins de celui du français.

Manuals such as “The Ladies' Grammar Book” were common in the 18th and early 19th centuries. Such a specification implies a concept (LADY) that refers to a social entity, which, in its turn, forms part of the ideology of a community at a certain moment in time. Setting out the history of "Ladies' Grammar Books" thus suggests an approach to grammar that takes into account socioideological considerations rather than theories and traditions of grammatical analysis. The main questions will be the following: What are the pertinent aspects of the socio-ideological angle? How can they be identified and integrated into an overview of the whole? How can they be interpreted in a larger historical and theoretical framework? The empirical section will analyse 25 “Ladies' Grammar Books" published between 1728 and 1845 (15 for the French, 10 for the Italian language). By reference to some of the parameters established in the course of the theoretical considerations it will be possible to study: to what degree the concrete grammar book corresponds to the theoretical suppositions, at which point it is possible to determine local traditions (France and Geneva compared to the German-speaking countries), at what point the status of the language (native or foreign) explains certain characteristics, in which sense the study of Italian suggests other contents and different paths from that of French. 
INDEX

Mots-clés : Histoire de la grammaire, Grammaire française, Grammaire italienne, Études sur l'identité de genre, Genre (gender) et enseignement des langues, Historiographie de la grammaire

Keywords : History of grammar, French grammar, Italian grammar, Gender studies, Gender and language teaching, Historiography of grammar

\section{AUTEUR}

\section{GABRIELE BECK-BUSSE}

Université de Marburg 